\title{
Changing scenario in genetics
}

\author{
A. K. Sharma
}

Published online: 7 June 2012

(C) Archana Sharma Foundation of Calcutta 2012

The outlook on the structure and modality of functioning of genes in chromosomes has undergone enormous modifications with technological advances in the past few decades. The major shift from the original concept, to start with, had been the demonstration of the split nature of the genes composed of essential (exon) and non essential (intron) sequences. The so called introns, the antiquity of which almost concomitant to metazoan evolution, along with the repeat sequences of varying nature, have played important roles in genome evolution. In the functioning of genes, the role of a variety of repeats, the transcription factors and regulators are well documented.

The relationship of gene and enzyme, basic for gene expression has assumed a new dimension than ever before. The demonstration of alternate splicing, along with normal splicing, joining of non-colinear exons - both intra and extra-chromosomal - chimeral transcripts have added to complexities in genome - transcriptome relationship. The repercussion on proteome as well, the latter being the total protein components of the organism encoded in the genome, is obvious.

Simultaneously, of the DNA sequences at the chromosome level, the mobile or transposable ones, because of their dynamicity, have come to the forefront. In addition to inducing mutations at insertion sites, their pleiotropic effects, effects on adjacent sequences, and their property of amplification, have become significant factors in evolutionary progress. Lately, a very high copy number of transposable sequences have been reported influencing to a great extent the genome evolution. The increase in genome size has also been reported to the range of 1 to 2.4 billion bases in maize. In fact, records of amplified gene sequences following transposition, contributing to ecological adaptations are frequent.

All these developments recorded periodically by different authors have led to deeper insight into the structure and functioning of genes as well as facets of chromosome dynamics, hitherto almost unknown.

The epigenetic modifications as mentioned in an earlier editorial [3], have provided with a new outlook. These modifications - DNA methylation, histone acetylation (e.g. Fig. 1), protein modification, non coding and siRNA, all forming a coating over the genome affect development to a remarkable degree. In fact, these events are also indicators to the basic fact as to why the widely different mammalian species differ so little in their genomic component. Their differences owe, to a great extent as claimed [1] to epigenetic mechanism, permitting similar DNA sequences expressing uniquely in different cells, at different periods and phases and at different environmental set up including micro environment.

Differential effects of epigenome in polyploids vis-à-vis diploids are also on record [2]. A detailed cataloguing of

A. K. Sharma $(\square)$

Department of Botany, Centre of Advanced Studies in Cell and

Chromosome Research, University of Calcutta,

Kolkata 700 019, India

e-mail: nuclaks@gmail.com 

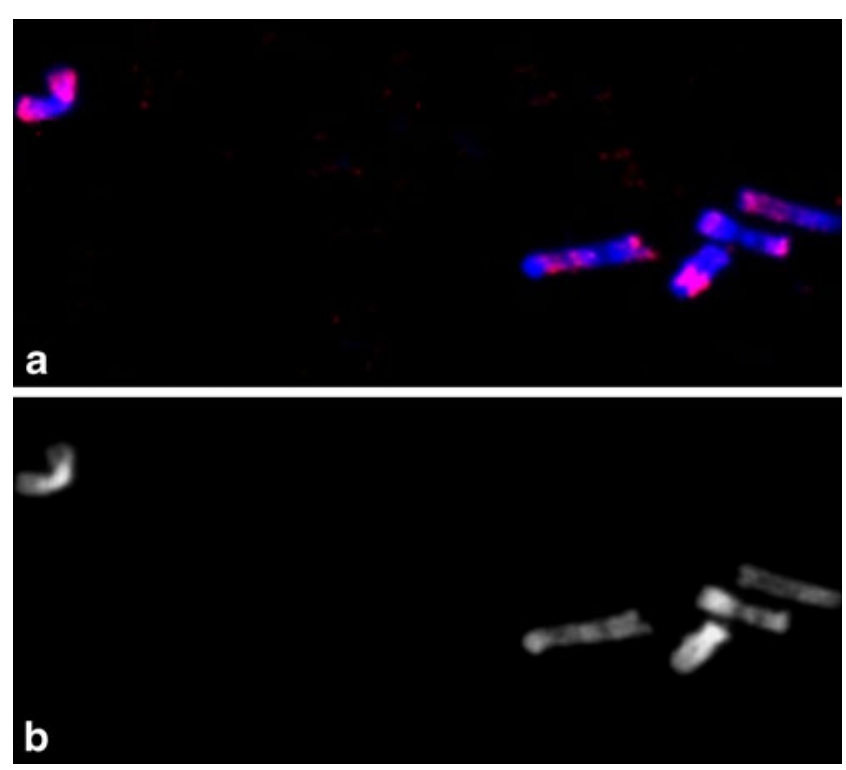

Fig. 1 Immuno-detection of histone modification on plant chromosomes: (a) red signals showing methylation marks detected by euchromatin methylation specific antibody $\mathrm{H} 3 \mathrm{~K} 4 \mathrm{me} 2$, (b) DAPI positive heterochromatic bands on the same (photograph by UC Lavania and Y. Mukai) epigenetic modifications and their implications is indeed called for. The IHEC (International Human Epigenome Consortium) is working worldwide to decipher precise epigenomic changes during the development and expression of genes. Once the mapping of entire epigenome - a genome wide map of epigenetic modifications - is completed along with the clarification of the extent of genomic control - a vision, which has recently been compared with that of Large Hadron Collider (LHC), the blueprint underpining the entire diversity of life and its challenges would be at hand with immense possibility.

\section{References}

1. Editorial. Time for the epigenome. Nature. 2010;463:587.

2. Lavania UC, Srivastava S, Lavania S, Basu S, Misra NK. Autopolyploidy differentially influences body size in plants, but facilitates enhanced accumulation of secondary metabolites, causing increased cytosine methylation. Plant J. 2012. doi:1111/j.1365313X.2012.05006.x.

3. Sharma AK. Genomics, epigenomics and chromatin. Nucleus. 2011;54:1-2. 